\title{
A Spitzer Survey for Dust-Obscured Supernovae
}

\author{
Ori D. Fox ${ }^{1,2}$, Harish Khandrika ${ }^{1}$, David Rubin ${ }^{3}$, Chadwick Casper ${ }^{4,5}$, \\ Gary Z. $\mathrm{Li}^{4,6}$, Tamás Szalai ${ }^{7,8}$, Lee Armus ${ }^{9}$, Alexei V. Filippenko ${ }^{4,10}$, \\ Michael F. Skrutskie ${ }^{11}$, Lou Strolger ${ }^{1}$, Schuyler D. Van Dyk ${ }^{9}$ \\ ${ }^{1}$ Space Telescope Science Institute, 3700 San Martin Drive, Baltimore, MD 21218, USA. \\ 2ofox@stsci.edu. \\ ${ }^{3}$ Department of Physics and Astronomy, University of Hawai ‘ $i$ at Mānoa, Honolulu, Hawai $i$ 96822, USA. \\ ${ }^{4}$ Department of Astronomy, University of California, Berkeley, CA 94720-3411, USA. \\ ${ }^{5}$ Glint Photonics, 1520 Gilbreth Rd., Burlingame, CA 94010, USA. \\ ${ }^{6}$ The Aerospace Corporation, 2310 E. El Segundo Blvd., El Segundo, CA 90245, USA. \\ ${ }^{7}$ Department of Optics and Quantum Electronics, University of Szeged, H-6720 Szeged, Dóm tér 9, Hungary. \\ ${ }^{8}$ Konkoly Observatory, Research Centre for Astronomy and Earth Sciences, H-1121 Budapest, Konkoly Thege Miklós út 15-17, Hungary. \\ ${ }^{9}$ IPAC, Caltech, 1200 E. California Blvd., Pasadena, CA 91125, USA. \\ ${ }^{10}$ Miller Institute for Basic Research in Science, University of California, Berkeley, CA 94720, USA. \\ ${ }^{11}$ Department of Astronomy, P.O. Box 3818, University of Virginia, Charlottesville, VA 22903-0818, USA.
}

13 July 2021

\begin{abstract}
Supernova (SN) rates serve as an important probe of star-formation models and initial mass functions. Near-infrared seeing-limited ground-based surveys typically discover a factor of 3-10 fewer SNe than predicted from far-infrared (FIR) luminosities owing to sensitivity limitations arising from both a variable point-spread function (PSF) and high dust extinction in the nuclear regions of star-forming galaxies. This inconsistency has potential implications for our understanding of star-formation rates and massive-star evolution, particularly at higher redshifts, where star-forming galaxies are more common. To resolve this inconsistency, a successful SN survey in the local universe must be conducted at longer wavelengths and with a space-based telescope, which has a stable PSF to reduce the necessity for any subtraction algorithms and thus residuals. Here we report on a two-year Spitzer/IRAC $3.6 \mu \mathrm{m}$ survey for dust-extinguished $\mathrm{SNe}$ in the nuclear regions of forty luminous infrared galaxies (LIRGs) within $200 \mathrm{Mpc}$. The asymmetric Spitzer PSF results in worse than expected subtraction residuals when implementing standard template subtraction. Forward-modeling techniques improve our sensitivity by several 1.5 magnitudes. We report the detection of $9 \mathrm{SNe}$, five of which were not discovered by optical surveys. After adjusting our predicted rates to account for the sensitivity of our survey, we find that the number of detections is consistent with the models. While this search is nonetheless hampered by a difficult-to-model PSF and the relatively poor resolution of Spitzer, it will benefit from future missions, such as Roman and JWST, with higher resolution and more symmetric PSFs.
\end{abstract}

Key words: supernovae: general — dust, extinction — infrared: stars

\section{INTRODUCTION}

The observed rate at which stars more massive than $\sim 8 \mathrm{M}_{\odot}$ explode as core-collapse supernovae (CCSNe) can be used to determine chemical evolution and feedback processes (Matteucci et al. 2006; Scannapieco \& Bildsten 2005), progenitor-mass distributions (Smith et al. 2011), star-formation rates (Iben 1983; Mannucci et al. 2007), and dust yields (Maiolino et al. 2004a,b; Rho et al. 2009). Given the intrinsic brightness of SNe, they serve as useful probes of the above characteristics at higher redshifts where other techniques are less feasible (e.g., Dahlén \& Fransson 1999; Dahlen et al. 2004; Strolger et al. 2015).

SN rates are useful probes, however, only if we understand the models linking the initial mass function (IMF), star-formation rates (SFRs), and SNe. For example, Mattila \& Meikle (2001) derive the expected CCSN rate (CCSNr) as a function of a galaxy's far-infrared (FIR) luminosity, $L_{\text {FIR }}=L_{(8-1000)} \mu \mathrm{m}$ (which is used as a proxy for star formation):

$\mathrm{CCSNr}=\frac{2.7 L_{\mathrm{FIR}}}{10^{10} \mathrm{~L}_{\odot}} \mathrm{SN}(100 \mathrm{yr})^{-1}$. 


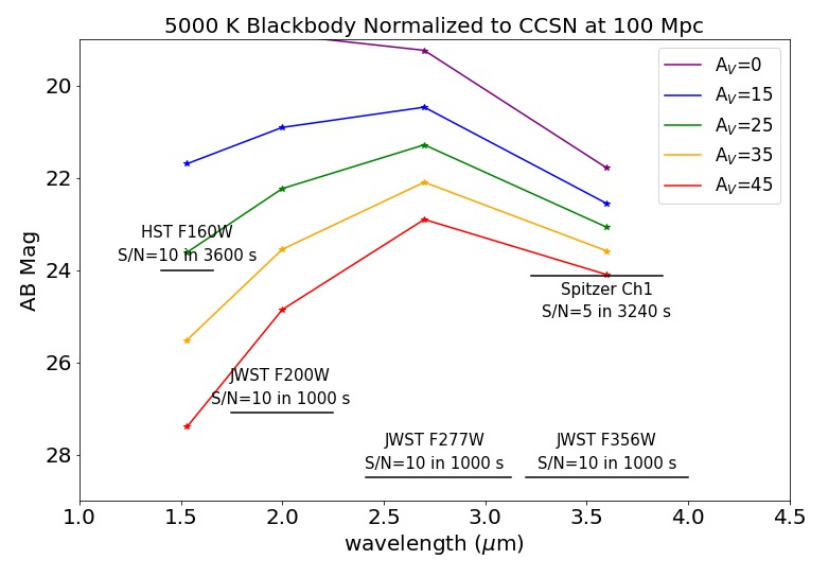

Figure 1. The effects of dust on the intensity and shape of a blackbody spectrum. The SED here is that of a $5000 \mathrm{~K}$ blackbody assumed to represent the photosphere of a SN IIP several months post-explosion. The $3.6 \mu \mathrm{m}$ flux is normalised to $M=-16 \mathrm{mag}$ Vega (see Fig. 2). Since more absorption occurs at shorter wavelengths, the peak of the spectrum shifts beyond the NIR (i.e., $2.6 \mu \mathrm{m}$ ) as $A_{V}$ increases beyond $25 \mathrm{mag}$. Spitzer is more efficient than $H S T$ at these higher extinction levels.

While Equation 1 is an empirical relationship, Mattila \& Meikle (2001) derive a similar relation (their Eq. 5) from first principles and show that Equation 1 both has a basis in and is consistent with the theoretical connection between the FIR luminosity and SFR. Mattila et al. (2012) add that this empirical relationship could have a significant uncertainty owing to a small sample size. We further note that the theoretically derived relationship also has significant uncertainty since it assumes a scaling factor between the intrinsic FIR-specific luminosity and the SFR, with no correction for extinction or other effects ${ }^{1}$, of about $\kappa_{\mathrm{FIR}} \approx 4 \times 10^{-10} \mathrm{M}_{\odot} \mathrm{yr}^{-1} \mathrm{~L}_{\odot}^{-1}$. This is about twice as large as the value determined for dusty galaxies by Madau \& Dickinson (2014) and Kennicutt (1998), at ages of above around $300 \mathrm{Myr}^{2}$ The derivation also assumes the fraction of stars ultimately successful in producing CCSNe is $k \approx 0.007 \mathrm{M}_{\odot}^{-1}$. This value is $\sim 30 \%$ larger than estimated for average galaxies by Strolger et al. (2015), but also carries some assumptions on progenitor mass ranges an/d average shape of the high-mass IMF. Considering the relationship between all of these variables, the observed CCSNr therefore gives a useful constraint on the model by providing some leverage on our theoretical understanding of $k$ and $\kappa_{\text {FIR }}$.

The ability to detect all CCSNe in a survey limits the completeness of any rate study. This is particularly true in galaxies with high SFRs, where gas and dust obscure many CCSNe. In fact, optical-wavelength surveys in dusty, star-forming (ultra)-luminous IR galaxies (LIRGs and ULIRGs) have routinely established CC$\mathrm{SNr}$ values that are unexpectedly similar to those in more normal galaxies (Richmond et al. 1998; Navasardyan et al. 2001). Strolger

\footnotetext{
1 This includes the an unknown efficiency in reprocessing UV radiation from stars into dust emission in the far-IR, which for starbursts with high optical depths is around $100 \%$.

2 Younger galaxies $(\lesssim 300 \mathrm{Myr}$ ) have higher emission-to-star-formation ratios as short-lived massive stars still dominate UV emission that is reprocessed and re-emitted in the IR.
}

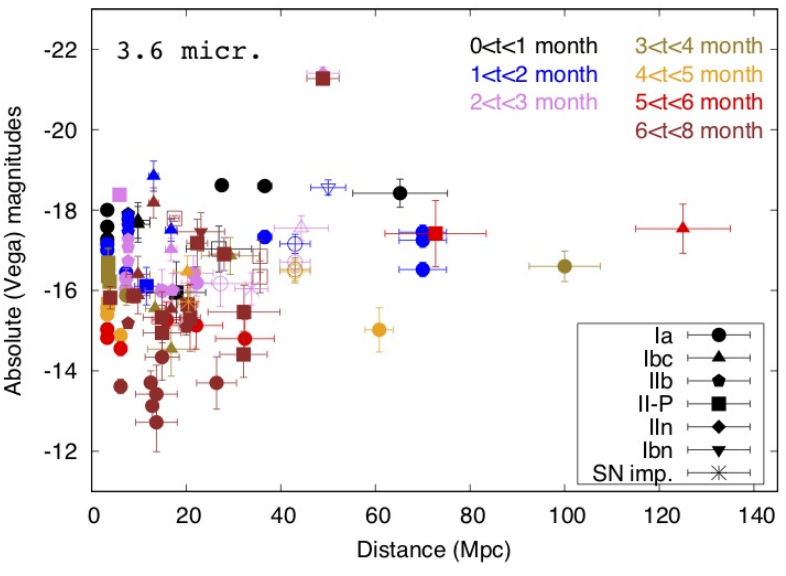

Figure 2. Early-time MIR evolution of all historical SNe obtained from Spitzer archival data by Szalai et al. (2019). Filled and open symbols denote SNe where photometry was obtained with or without image subtraction, respectively. SN subtypes (e.g., Filippenko 1997) are denoted in the legend, and "SN imp" refers to SN impostors.

et al. (2015) derive the CCSNr from the Hubble Space Telescope (HST) CANDELS and CLASH programs, two extragalactic multicycle treasury programs using the WFC3 IR channel with sensitivities down to $25.5 \mathrm{mag}_{\text {Vega }}$ out to $1.6 \mu \mathrm{m}$. The observations are notably different from the ultraviolet (UV)/optical SFR (Madau \& Dickinson 2014), and even less so with the mid-IR (MIR) and FIR predictions (Chary \& Elbaz 2001). Strolger et al. (2015) link the low CCSNr (and the subsequent source of discrepancy between the UV/optical and IR-derived SFRs) to dust obscuration, particularly in galaxies with high SFRs, where gas and dust obscure most of the $\mathrm{SNe}$, in agreement with Mattila et al. (2012).

The highest SFRs in the local Universe are found in LIRGs and ULIRGs $\left(L_{\text {FIR }}>10^{11} \mathrm{~L}_{\odot}\right.$ and $10^{12} \mathrm{~L}_{\odot}$, respectively; Sanders et al. 2003). Although one must account for the contribution from hidden active galactic nuclei (AGNs), star formation tends to contribute most of the IR luminosity (Sanders \& Mirabel 1996; Genzel et al. 1998), and estimates of the relative AGN power have been made using a variety of MIR tracers in hundreds of local IR galaxies (e.g., Díaz-Santos et al. 2017). (U)LIRGs account for only a small fraction of the local ( $<100 \mathrm{Mpc}$ ) galaxy population (Sanders et al. 2003), so obscuration in these galaxies does not have a detrimental effect on local CCSNr studies. At higher redshifts, however, obscured star formation in (U)LIRGs dominates over star formation traced by UV and optical light (e.g., Floc'h et al. 2005; Magnelli et al. 2009, 2011; Strolger et al. 2015). To accurately measure the CCSNr as a function of redshift, the local CCSNr in starburst galaxies, such as (U)LIRGs, must be fully characterised.

The IR, which can be up to 10 times less affected by extinction than visible light, offers an optimised window for SN searches in these starburst galaxies. Already, a number of near-IR (NIR) searches have been performed with mixed success. Early NIR ground-based surveys were hampered by poor resolution and limited telescope time (van Buren \& Greenhouse 1994; Grossan et al. 1999; Maiolino et al. 2002; Mannucci et al. 2003; Mattila et al. 2004; Mattila et al. 2005a,b; Miluzio et al. 2013). These studies resulted in an inferred CCSNr that was still a factor of 3-10 lower than expected from the SFR (Mannucci et al. 2003). Furthermore, no SNe were detected within the galaxy nuclei. Ground-based high-resolution adaptive optics (AO) NIR searches have had some additional success in their discovery of several CCSNe within $<300 \mathrm{pc}$ from the 
Table 1. Target Galaxies

\begin{tabular}{|c|c|c|c|c|c|c|c|}
\hline Name & RA (J2000) & Dec (J2000) & $\begin{array}{r}\text { Distance } \\
(\mathrm{Mpc})\end{array}$ & $\begin{array}{r}L_{\mathrm{FIR}} \\
\left(\log \mathrm{L}_{\odot}\right)\end{array}$ & \# of SNe & $\begin{array}{r}\text { \# of SNe } \\
\text { (Corrected) }\end{array}$ & Detections \\
\hline NGC 34 & 00:11:06.612 & $-12: 06: 28.33$ & 83.33 & 11.43 & 1.45 & 0.42 & 0 \\
\hline NGC 232 & $00: 42: 45.814$ & $-23: 33: 40.69$ & 91.66 & 11.23 & 0.92 & 0.28 & 0 \\
\hline MCG+12-02-001 & 00:54:03.943 & $+73: 05: 05.23$ & 66.67 & 11.29 & 1.05 & 0.71 & 0 \\
\hline IC 1623 & 01:07:47.2 & $-17: 30: 25$ & 79.17 & 11.38 & 1.30 & 0.35 & 0 \\
\hline UGC 2369 & 02:54:00.9 & $+14: 58: 31$ & 129.17 & 11.44 & 1.49 & 0.09 & 0 \\
\hline IRAS $03359+1523$ & 03:38:46.9 & $+15: 32: 55$ & 145.83 & 11.38 & 1.30 & 0.04 & 1 \\
\hline MCG-03-12-002 & $04: 21: 20.0$ & $-18: 48: 45$ & 133.33 & 11.30 & 1.08 & 0.05 & 0 \\
\hline NGC 1572 & $04: 22: 42.814$ & $-40: 36: 03.50$ & 83.33 & 11.16 & 0.78 & 0.34 & 0 \\
\hline NGC 1614 & 04:34:00.027 & $-08: 34: 44.57$ & 66.67 & 11.41 & 1.39 & 0.56 & 0 \\
\hline NGC 2623 & 08:38:24.093 & $+25: 45: 16.70$ & 75.00 & 11.47 & 1.59 & 0.82 & 0 \\
\hline UGC 4881 & 09:15:55.54 & $+44: 19: 58.2$ & 166.67 & 11.57 & 2.01 & 0.01 & 0 \\
\hline UGC 5101 & 09:35:51.694 & $+61: 21: 10.52$ & 162.50 & 11.90 & 4.29 & 0.02 & 0 \\
\hline MCG+08-18-012 & 09:36:30.7 & $+48.28: 10$ & 108.33 & 11.19 & 0.84 & 0.14 & 0 \\
\hline IC 563/IC 564 & 09:46:20.361 & $+03: 02: 43.86$ & 83.33 & 11.10 & 0.68 & 0.29 & 1 \\
\hline NGC 3110 & 10:04:02.124 & $-06: 28: 29.12$ & 66.67 & 11.10 & 0.68 & 0.44 & 0 \\
\hline NGC 3256 & $10: 27: 51.60$ & $-43: 54: 18.0$ & 37.50 & 11.44 & 1.49 & 1.25 & 1 \\
\hline IRAS $10565+2448$ & $10: 59: 18.153$ & $+24: 32: 34.30$ & 175.00 & 11.87 & 4.00 & 0.03 & 0 \\
\hline Arp 148 & 11:03:53.892 & $+40: 50: 59.89$ & 141.67 & 11.50 & 1.71 & 0.06 & 1 \\
\hline MCG+00-29-0023 & 11:21:12.261 & $-02: 59: 03.00$ & 100.00 & 11.36 & 1.24 & 0.26 & 0 \\
\hline IC 2810/UGC 6436 & $11: 25: 45.055$ & $+14: 40: 35.98$ & 141.67 & 11.50 & 1.71 & 0.06 & 0 \\
\hline NGC 3690 & $11: 28: 33.13$ & $+58: 33: 58.0$ & 45.83 & 11.72 & 2.83 & 0.24 & 0 \\
\hline ESO507-G070 & $13: 02: 52.354$ & $-23: 55: 17.65$ & 87.50 & 11.31 & 1.10 & 0.39 & 0 \\
\hline UGC 8335 & $13: 15: 32.8$ & $+62: 07: 37$ & 129.17 & 11.60 & 2.15 & 0.06 & 0 \\
\hline UGC 8387 & 13:20:35.380 & $+34: 08: 21.84$ & 95.83 & 11.52 & 1.79 & 0.23 & 0 \\
\hline NGC 5256 & 13:38:17.69 & $+48: 16: 33.9$ & 112.50 & 11.37 & 1.27 & 0.16 & 0 \\
\hline NGC 5257 & $13: 39: 52.273$ & $+00: 50: 22.48$ & 91.67 & 11.37 & 1.27 & 0.40 & 1 \\
\hline Mk 273 & $13: 44: 42.070$ & $+55: 53: 13.17$ & 158.33 & 12.10 & 6.80 & 0.08 & 0 \\
\hline NGC 5331 & 13:52:16.15 & $+02: 06: 03.3$ & 137.50 & 11.43 & 1.45 & 0.06 & 0 \\
\hline UGC 8782 & $13: 52: 17.7$ & $+31: 26: 44$ & 187.50 & 12.27 & 10.06 & 0.00 & 0 \\
\hline Arp 302 & $14: 57: 00.4$ & $+24: 36: 44$ & 141.67 & 11.59 & 2.10 & 0.05 & 0 \\
\hline Mk 848 & $15: 18: 06.123$ & $+42: 44: 44.59$ & 166.67 & 11.72 & 2.83 & 0.03 & 0 \\
\hline Arp 220 & $15: 34: 57.272$ & $+23: 30: 10.48$ & 75.00 & 12.12 & 7.12 & 3.52 & 0 \\
\hline NGC 6090 & 16:11:40.39 & $+52: 27: 21.5$ & 120.83 & 11.35 & 1.21 & 0.11 & 1 \\
\hline NGC 6240 & 16:52:58.97 & $+02: 24: 01.7$ & 100.00 & 11.85 & 3.82 & 0.65 & 2 \\
\hline IRAS 17208-0014 & $17: 23: 21.943$ & $-00: 17: 00.96$ & 179.17 & 12.30 & 10.77 & 0.05 & 0 \\
\hline IC 4687/86 & 18:13:39.829 & $-57: 43: 31.25$ & 70.83 & 11.35 & 1.21 & 0.68 & 0 \\
\hline IRAS $18293-3413$ & $18: 32: 40.2$ & $-34: 11: 26$ & 75.00 & 11.63 & 2.30 & 0.97 & 1 \\
\hline NGC 6926 & 20:33:06.108 & $-02: 01: 39.07$ & 83.33 & 11.11 & 0.70 & 0.29 & 0 \\
\hline NGC 7130 & 21:48:19.490 & $-34: 57: 04.73$ & 66.67 & 11.21 & 0.88 & 0.48 & 0 \\
\hline IRAS 23128-5919 & 23:15:46.772 & $-59: 03: 15.94$ & 183.33 & 11.80 & 3.41 & 0.01 & 0 \\
\hline
\end{tabular}

LIRG nuclei (e.g. Mattila et al. 2007; Kankare et al. 2008, 2012; Kool et al. 2018; Kankare et al. 2021; Pérez-Torres et al. 2021). Kool et al. (2018) note their high-spatial-resolution search uncovered a larger concentration of nuclear $\mathrm{SNe}$ in (U)LIRGs than previous low-resolution searches, while Kankare et al. (2021) showed that a sample of $29 \mathrm{CCSNe}$ at $<2.5 \mathrm{kpc}$ from (U)LIRG nuclei showed a correlation between the startburst age and SN subtype. In general, these gorund-based surveys either did not find their rates consistent with the predictions or had limited statistics. The overall conclusion was that a majority of SNe likely occur within the nuclei, but the extinction is so high $\left(A_{V}>25 \mathrm{mag}\right)$ and the nuclei are so bright that longer-wavelength observations are necessary. Even several space-based surveys (e.g., Bregman et al. 2000), including a HST/NICMOS (Cresci et al. 2007), turned up no confirmed detections, concluding that the ULIRG dust-extinction values are too high $\left(A_{V}>25 \mathrm{mag}\right)$. Results from the SPitzer InfraRed Intensive Transients Survey (SPIRITS; Kasliwal et al. 2017) between 2014 and 2018 find the fraction of CCSNe in nearby galaxies missed by optical surveys could be as high as $38.5 \%$ (Jencson et al. 2019). High-resolution ground-based radio surveys, by contrast, have suc- cessfully discovered nuclear $\mathrm{SNe}$ with rates consistent with the galaxy IR luminosities, but not all $\mathrm{SNe}$ are sufficiently bright at radio wavelengths to conduct a complete survey (Pérez-Torres et al. 2021).

Until the James Webb Space Telescope (JWST) is launched, the Spitzer Space Telescope (Spitzer) Infrared Array Camera (IRAC; Fazio et al. 2004) offered the best combination of long-wavelength sensitivity, stable point-spread function (PSF), moderate resolution, and sensitivity to $\mathrm{SN}$ radiation. Here we describe a post-cryogenic Warm-Spitzer/IRAC survey conducted in the years 2012-2014 for dust-extinguished $\mathrm{SNe}$ in, but not limited to, the nuclear regions of nearby star-forming (U)LIRGs (PID 90031; PI: O. Fox). (A similar, but more limited survey was conducted during the cryogenic Spitzer mission by Lawrence \& Gautier 2004, PID 108; however, the data analysis and results were never published.) The MIR is optimised for dust-extinction levels $A_{V}>25 \mathrm{mag}$. The improved sensitivity offers an improved estimate of the number of SNe missed by visible (and NIR) surveys and tighter constraints on our understanding of star-formation models out to high redshifts. The direct product of this study is the connection between FIR luminosity and massive- 

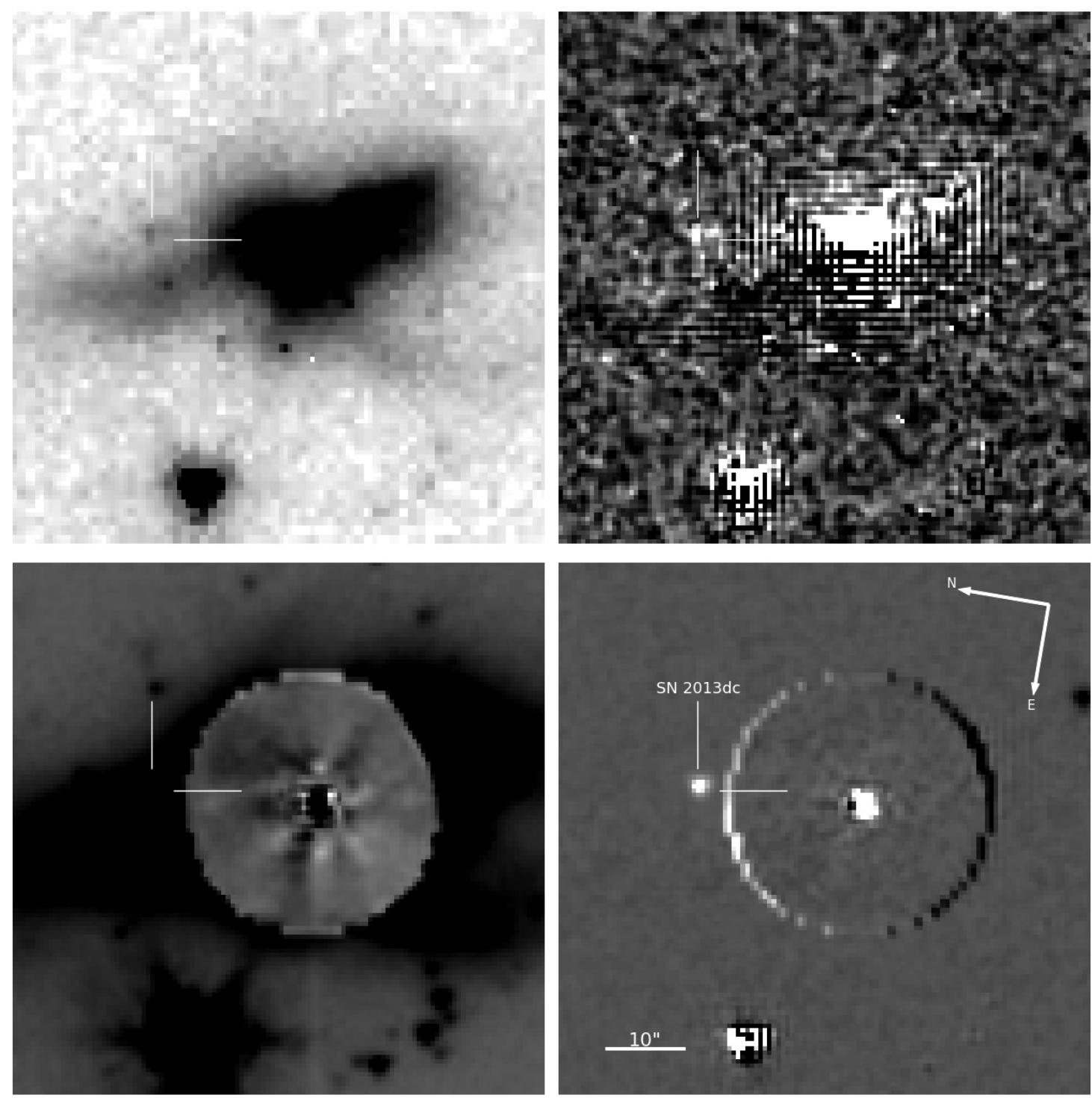

Figure 3. Comparison of sample data products, in this case for the first epoch of NGC 6240, used in our transient search. (Top left:) Original, unsubtracted MOPEX processed data. (Top right:) Standard alignment and subtraction of two epochs. (Bottom left:) Forward modeling subtraction, where the circle corresponds to our forward modeling radius. (Bottom right:) Subtraction of two epochs that have been forward modeled, but in this case are spaced nearly one year apart so that the telescope roll angle is similar. The colour scale and stretch is constant for all subtracted images shown in this figure. The point source is SN 2013dc. It is present in all the images, but is most clearly detected in the bottom right. The central source is the galaxy nucleus. As described in the text, there are a number of caveats for modeling the inner core, including nonlinearity concerns. Correcting those issues is beyond the scope of this paper, but we account for them in our statistics.

star formation. Such results naturally connect to the high-redshift universe, where a fundamental observable is the FIR galactic flux. Given a future which includes the LSST, EUCLID, the Nancy Grace Roman Space Telescope (NGRST), and JWST, local measurements characterising the properties of galaxies selected from FIR samples have large-scale implications.

Our observations are presented in Section 2. Section 3 describes the data reduction, data processing, and analysis techniques. Our results are discussed in Section 4, and Section 5 summarises our conclusions.

\section{OBSERVATIONS}

The original proposal called for a Warm-Spitzer/IRAC survey of 40 galaxies to maximise the number of expected $\mathrm{SNe}$ and generate a statistically significant sample to minimise the statistical effects of undetected SNe. We selected the nearest 40 (U)LIRGs $\left(L_{\mathrm{FIR}}>\right.$ $10^{10} \mathrm{~L}_{\odot}$ ) from Sanders et al. (2003), which ultimately extended our sample out to $200 \mathrm{Mpc}$. Table 1 summarises the targets and also lists the number of SNe predicted by Equation 1 over the two-year survey window. For each galaxy, eight epochs of observations were obtained, spaced approximately 1-6 months apart depending on the visibility window.

To minimise observing time, all observations were obtained in only a single filter with IRAC Channel $1(3.6 \mu \mathrm{m})$. The spectral en- 


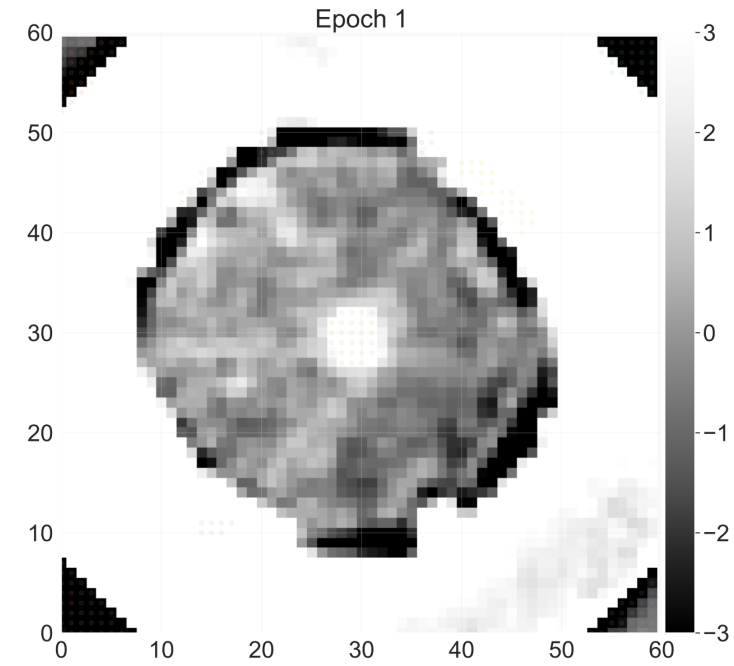

Figure 4. A residual map of UGC 8782 illustrating complications and limitations of our algorithm to detect interconnected groups of pixels within a given epoch with fluxes $\gtrsim 3 \sigma$ of their average value. The original image has the forward model subtracted, resulting in the ringed structure corresponding to the model itself. The color scale units are $\sigma$, defined by the statistics of each individual pixel across all eight epochs. The poorly subtracted nuclear region in this example is typical of many of the targets in our sample for a variety of issues beyond forward modeling that we describe and account for in the text.

ergy distribution (SED) for a $5000 \mathrm{~K}$ blackbody peaks at $>2.0 \mu \mathrm{m}$ for $A_{V}>25 \mathrm{mag}$ (Fig. 1). Increasing the extinction pushes the peak of the SED to longer wavelengths, but also lowers the overall flux substantially enough that it becomes inefficient to observe these targets at larger distances. Channel $1(3.6 \mu \mathrm{m})$ is also slightly more sensitive than Channel $2(4.5 \mu \mathrm{m})$. These are the only two channels available during the Warm-Spitzer mission and, at the time of the survey, Spitzer/IRAC was the only MIR observing capabilities accessible from space.

To calculate our integration times, we consider the distribution of CCSN magnitudes. MIR light curves of CCSNe are sparse, but Szalai et al. (2019) present comprehensive plots of all existing Spitzer observations. Figures 6 and 7 from Szalai et al. (2019) show that CCSNe plateau in the MIR for $\sim 300$ days post-explosion, and often even longer. Figure 2 plots the photometry as a function of distance, separated by explosion type and age. A majority of CCSNe fall within a range of $-16<M_{3.6 \mu m}<-18$ over the first few months. Most of these $\mathrm{SNe}$ are at distances $<60 \mathrm{Mpc}$, since the proposed Spitzer observations generally targeted nearby, bright SNe.

Given that the typical time between consecutive observations is anywhere in the range 1-6 months, we assumed a magnitude of $M_{3.6 \mu \mathrm{m}} \approx-17$ when designing our observations. In general, photon-counting statistics from the underlying galaxy dominate the uncertainty, and we therefore require longer integration times. We also account for possible extinction ranging up to $A_{V}=35 \mathrm{mag}$. Figure 1 shows the effects of extinction by plotting the SED for a $5000 \mathrm{~K}$ blackbody for various values of $A_{V}$. At $3.6 \mu \mathrm{m}$, the effects of extinction are minimal, decreasing the brightness only $\sim 2$ mag for $A_{V}=45 \mathrm{mag}$. Despite this planning, however, subtraction effects ultimately dominated our error budget, which we discuss in more detail below.

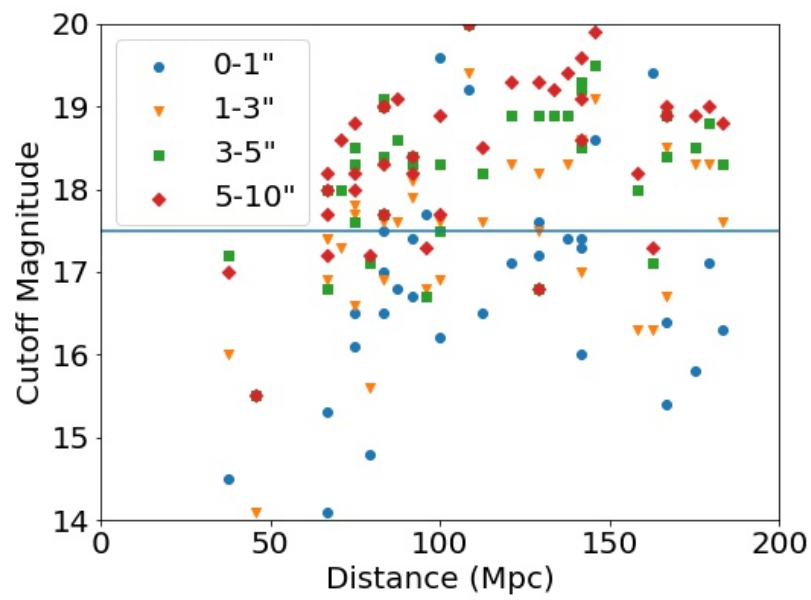

Figure 5. Sensitivity of our search algorithm derived from the artificial source tests for each galaxy as a function of the galaxy distance. Each galaxy in our sample has four data points, corresponding to recovery rates for artificial sources placed at various radii from the nucleus as defined by the legend. The detection limit, or cutoff magnitude, is defined as the magnitude at which we can achieve a $50 \%$ recovery rate for all of our sources. The solid line corresponds to the more realistic empirical cutoff magnitude derived qualitatively from Figure 10, as we discuss in the text. Figure 6 plots the consolidated results for all galaxies shown in this plot.

\section{REDUCTION TECHNIQUES}

\subsection{Forward Modeling and Subtraction}

Despite the absence of atmospheric effects when working with images taken by space telescopes, there are three features of the Spitzer data that combine to make precise galaxy subtraction difficult. (1) The PSF is undersampled (only $\sim 1$ pixel per full width at halfmaximum intensity). (2) The PSF is highly azimuthally asymmetric. (3) The data are taken over a range of spacecraft roll angles, with no two epochs matching exactly. To enable precise galaxy subtraction, we turn to forward modeling.

Forward modeling infers an analytic galaxy model on the sky by tracing the light ("forward") through convolution with the PSF and pixel, and then sampling by the detector (e.g., Holtzman et al. 2008; Suzuki et al. 2012; Hayden et al. 2021; Rubin et al. 2021). Each of the epochs is fit simultaneously with a model of the galaxy background plus SN. This model is inferred using a set of dithers and/or rotations, bypassing the traditional step of resampling the images for pixel-by-pixel subtraction, which would introduce artifacts. In testing, we found that the given model of the Point Response Function $^{3}$ (PRF, the covolution of the PSF and the pixel response) was not accurate enough for our purposes, so we derived our own PRF from high signal-to-noise-ratio observations of field stars.

For each of the eight epochs in which a search is performed, the forward model is derived from reference observations of the galaxy obtained before the SN appears or after the SN fades. In other words, we exclude observations obtained $<20$ days prior or $>330$ days to account for the possibility that a rising/falling SN component may contribute to the model. In addition to the galaxy model, we also fit small tweaks to the astrometry and differences

\footnotetext{
${ }^{3}$ https://irsa.ipac.caltech.edu/data/SPITZER/docs/irac/calibrationfiles/psfprf/
} 


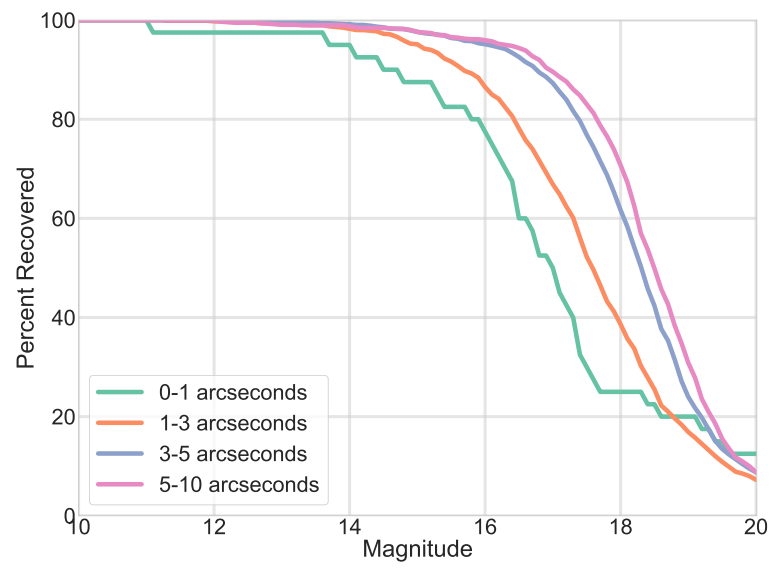

Figure 6. Overall detection rate as a function of radius from the galactic nucleus for forward model subtracted images based on artificial star tests

in the sky level. Once a model is generated, it is subtracted from each corrected Basic Calibrated Data (cBCD) file, which are then stacked with the MOsaicker and Point source EXtractor (MOPEX) ${ }^{4}$ (Fig. 3).

\subsection{Detecting Transient Sources}

To quantify the success of the forward-modeling algorithm, we use grids of artificial star tests. Owing to the IRAC mosaic's large pixel scale $\left(\sim 0.6^{\prime \prime}\right)$, however, the flux density within any single pixel tends to vary from epoch to epoch, particularly in rapidly changing (i.e., nuclear) environments. This could lead to false positives. each pixel over all eight epochs and then searches for a cluster of interconnected pixels within a given epoch that are all $>3 \sigma$ from their average value (e.g., Fig. 4). For each galaxy, we calculate the percent of SNe recovered using this method as a function of magnitude and distance from the centre of the galaxy. The detection limit, or cutoff magnitude, is defined as the magnitude at which we can achieve a $50 \%$ recovery rate and varies significantly depending on the individual features of the host galaxy (Fig. 5).

Figures 6 and 7 consolidate the results from Figure 5 and show the evolution of the detection rate as a function of magnitude for the different radii bins. Generally, the detection limit improves at larger radii owing to less complicated subtraction residuals. For comparison, results for the traditional (i.e., non-forward modeling) subtraction images are also plotted in Figure 7. Note how the forward modeling improves our relative fraction of faint $\mathrm{SNe}(>14 \mathrm{mag}$ ) recovered.

Using this $3 \sigma$ interconnected pixel detection algorithm, however, is limited given only eight epochs of data per pixel and the fact that $\mathrm{SNe}$ can span multiple epochs, thereby influencing the statistics of the pixels that we are trying to isolate. Any single threshold does not equally apply to all pixels because the asymmetric PSF impacts the noise in the model subtractions of some pixels in a non-Gaussian way. We also notice a subtle nonlinearity in the pixel response that We therefore developed an approach that considers the variance in

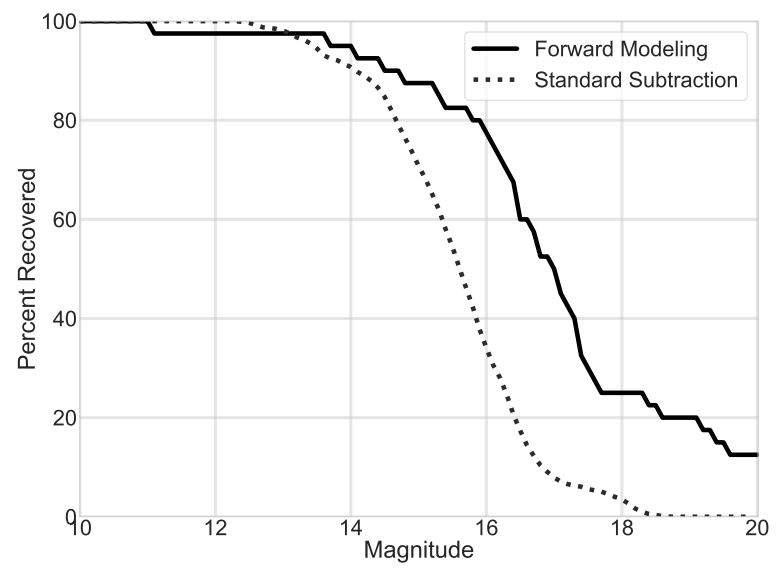

Figure 7. Overall detection rate for $0-5$ " comparing the standard and forward model subtraction techniques based on artificial star tests

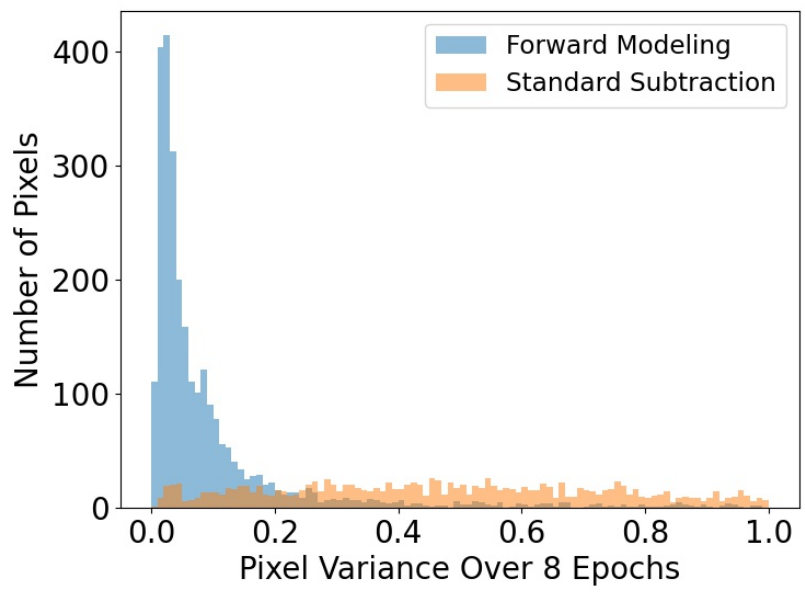

Figure 8. Histograms of flux-density variance per pixel in the inner $<3$ " of each galaxy using the two different reduction techniques: standard template subtraction and forward modeling. The histogram for the forward modeling is much narrower, suggesting less variance per pixel and overall increased sensitivity to detecting fainter sources.

evolves over the two-year lifetime of the survey (i.e., later epochs have lower fluxes). Pixels may therefore exhibit $3 \sigma$ deviations that are not due to Poisson statistics alone (Fig. 4). Furthermore, we have prior knowledge of the artificial source locations, so recovering the sources is not hampered with false positives or nearby noise effects.

All of this is to say that false positives could impact our interpretation of Figures 6 and 7. To alleviate any of these concerns, Figure 8 plots the distribution of variance in each pixel for the two different subtraction techniques. The forward-modeling approach has a much narrower distribution, which is consistent with an improved subtraction approach. We conclude that the artificial star tests are most useful for guiding the relative success of our forward modeling and subtraction algorithms, but the limitations of ${ }^{4}$ https://irsa.ipac.caltech.edu/data/SPITZER/docs/dataanalysistools/tools/mopex/ the simulations are significant enough that we cannot use them 
Table 2. Supernova Detections in this Survey

\begin{tabular}{|c|c|c|c|c|c|c|}
\hline $\begin{array}{l}\text { Host } \\
\text { Galaxy }\end{array}$ & $\begin{array}{l}\text { Source RA/Dec } \\
\text { (J2000) }\end{array}$ & $\begin{array}{l}\text { Nuclear Offset } \\
\left({ }^{\prime \prime N / E)}\right.\end{array}$ & $\begin{array}{l}\text { Date } \\
\text { (MJD) }\end{array}$ & $\begin{array}{c}F_{v,[3.6 \mu \mathrm{m}]} \\
(\mu \mathrm{Jy})\end{array}$ & $\begin{array}{c}m / M \\
(3.6 \mu m)\end{array}$ & Notes \\
\hline NGC 5257 & $13: 39: 52.2148,+0: 50: 25.328$ & $-11.8 / 0.3$ & 56780 & $12(5)$ & $18.3 /-16.5(0.5)$ & - \\
\hline IRAS $18293-3413$ & $18: 32: 41.0980,-34: 11: 27.230$ & $-1.0 / 0.2$ & $\begin{array}{l}56455 \\
56483 \\
56613 \\
56646\end{array}$ & $\begin{array}{l}55(5) \\
26(5) \\
87(5) \\
45(5)\end{array}$ & $\begin{array}{l}16.8 /-17.6(0.1) \\
17.6 /-16.8(0.3) \\
16.3 /-18.1(0.1) \\
17.0 /-17.4(0.2)\end{array}$ & SN 2013if \\
\hline Arp 148 & $11: 03: 53.1180,+40: 51: 09.385$ & $-8.8 / 9.5$ & 56510 & $1(0)$ & $21.2 /-14.6(0.1)$ & - \\
\hline NGC 6090 & $16: 11: 40.3172,+52: 27: 23.609$ & $-3.7 /-3.3$ & 56890 & $44(5)$ & $17.0 /-18.4(0.2)$ & - \\
\hline NGC 3256 & $10: 27: 50.7987,-43: 54: 02.554$ & $-5.2 / 11.0$ & $\begin{array}{l}56875 \\
56925\end{array}$ & $\begin{array}{l}610(10) \\
142(20)\end{array}$ & $\begin{array}{l}14.1 /-18.7(0.1) \\
15.7 /-17.1(0.2)\end{array}$ & PSN J10275082-4354034 \\
\hline IRAS 03359 & $03: 38: 47.3486,+15: 32: 56.462$ & $3.0 / 2.7$ & 56964 & $24(2)$ & $17.7 /-18.1(0.1)$ & - \\
\hline IC 563 & 09:46:20.7408, +3:02:38.346 & $5.7 /-5.5$ & 56687 & $31(3)$ & $17.4 /-17.2(0.3)$ & - \\
\hline NGC 6240 & $16: 52: 57.6161,+02: 23: 36.731$ & $-18.7 /-26.8$ & $\begin{array}{l}56795 \\
56833 \\
56953\end{array}$ & $\begin{array}{c}46(4) \\
20(5) \\
5(2)\end{array}$ & $\begin{array}{l}17.0 /-18.0(0.3) \\
17.8 /-17.2(0.4) \\
19.4 /-15.6(0.8)\end{array}$ & PS1-14xw \\
\hline NGC 6240 & $16: 52: 58.9791,+02: 24: 25.587$ & $1.8 / 22.0$ & $\begin{array}{l}56421 \\
56454 \\
56580 \\
56617\end{array}$ & $\begin{array}{l}56(5) \\
55(5) \\
8(2) \\
7(2)\end{array}$ & $\begin{array}{l}16.8 /-18.2(0.1) \\
16.8 /-18.2(0.1) \\
18.9 /-16.1(0.2) \\
19.0 /-16.0(0.2)\end{array}$ & SN 2013dc \\
\hline
\end{tabular}

as an absolute quantification of the sensitivity of our search. Ultimately, we choose to manually search for transient sources in the resulting images with limited guidance from our algorithm.

\section{RESULTS}

\subsection{Detections}

Table 2 lists, and Figures 9 and 10 show, all 9 detections from our sample. We also indicate any corresponding ground-based discoveries, which help us constrain the explosion dates. SN 2013if was discovered by the Supernova UNmasked By Infra-Red Detection (SUNBIRD) project and had an associated near-IR light curve (Kool et al. 2018). The best-fit light-curve template shows SN 2013if to be an SN IIP with almost no extinction, which is surprising given its proximity to the nucleus. Kankare et al. (2021) reported the discovery of PSN J10275082-4354034 near the nucleus of NGC 3256. The SN was also detected in serendipitous Hubble Space Telescope high-resolution imaging. The subsequent light curve was determined to be most consistent with an SN IIn, although an SN IIP could not be ruled out. Again, only a small amount of extinction ( $A_{V} \lesssim 0.3 \mathrm{mag}$ ) is present. PS1-14xw was first reported by Benitez et al. (2014) and classified as an SN Ia, while SN 2013dc was first reported by Block et al. (2013). We find no published analysis of either PS1-14xw or SN 2013dc.

Figure 11 plots the MIR light curves along with previously published light curves from Szalai et al. (2019). The MIR evolution of SNe Ia and SNe IIP overlap substantially, especially at early times, but they do tend to diverge a bit more at later times. Of the light curves that extend out to later epochs, the evolution is most consistent with SNe IIP. Of course, PS1-14xw was originally classified as an SN Ia. For the $\mathrm{SNe}$ for which we only have a single epoch, the brightness is consistent with both SNe Ia and SNe IIP around peak with very little to no extinction. The detection in Arp148 stands out for being relatively faint, which could indicate significant extinction or perhaps a poor constraint on the explosion time. It is difficult to draw any conclusions on the extinction from just a single filter.

Even with the forward modeling, all of our SN detections are outside $\left(>3^{\prime \prime}\right.$ ) of the galactic nucleus. (SN 2013if is a nuclear event included in our tally for rates purposes, but we do not have a convincing detection in Figure 9.) The survey has limited followup observations to confirm the nature and classification of each transient source, but the observed magnitudes are consistent with those of other SNe (Fig. 10), bright enough to rule out most other types of transients (see Fig. 4 of Kasliwal et al. 2017). For the purposes of this analysis, we assume that each detection (except PS1-14xw) is a CCSN.

\subsection{Survey Sensitivity}

Before we can interpret the statistics of our detections, it is important to have an understanding of our survey's sensitivity. We first consider our search sensitivity from an empirical perspective. Figure 10 plots the photometry of SNe detected in our survey (green points), as well as the relatively early $(<3$ month) magnitudes of the nearby $(<50 \mathrm{Mpc}) \mathrm{CCSNe}$ from Figure 2 (black points) and some young SNe Ia from Szalai et al. (2019) (red points). Overplotted are lines of constant apparent magnitude. The early-time magnitude distribution of nearby CCSNe is $-16 \lesssim M_{3.6 \mu m} \lesssim-18$, while known SNe Ia are a couple of magnitudes brighter. We assume that the nearby distribution of magnitudes is relatively complete. If our survey were also to be considered complete and the magnitude distribution doesn't change as a function of redshift, then to first order we should detect a similar spread in our $\mathrm{SN}$ magnitude distribution out to $200 \mathrm{Mpc}$.

The actual distribution of our SN photometry in Figure 10, however, does not reflect the more nearby distribution. Most of the 


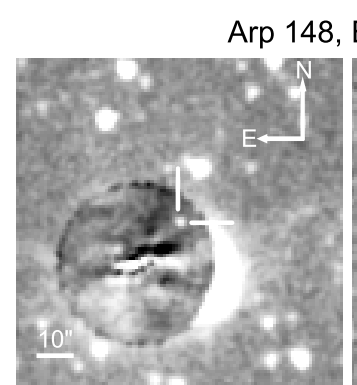

IRAS 03359+1523W, Epoch 7

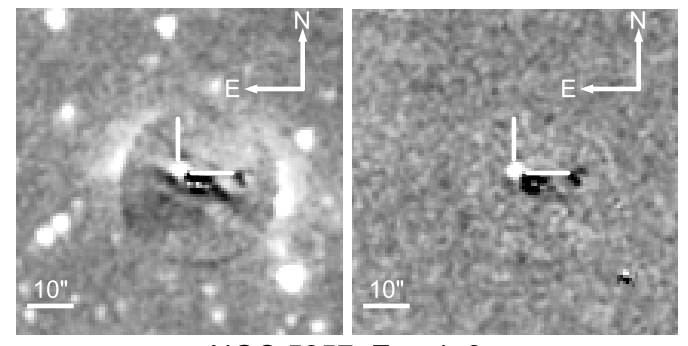

NGC 5257, Epoch 2
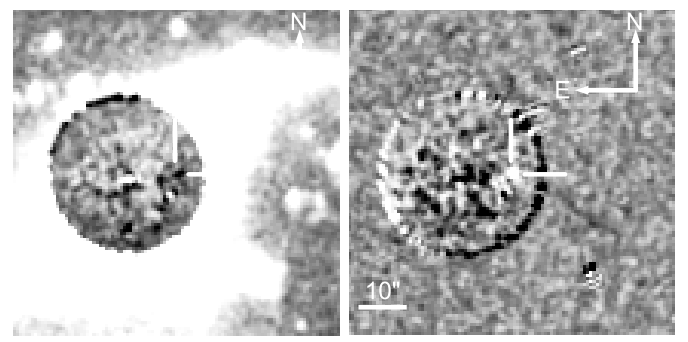

NGC 6240, Epoch 1

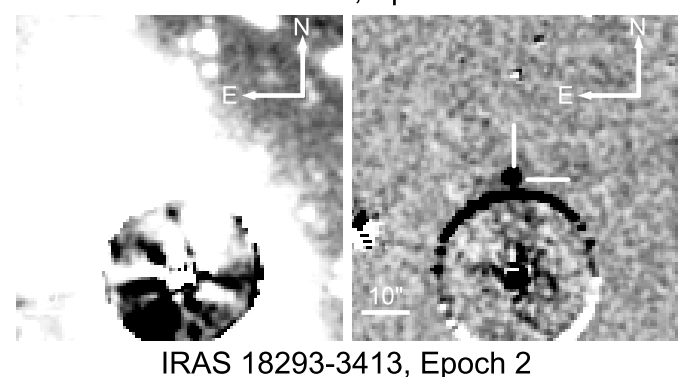

IRAS 18293-3413, Epoch 2

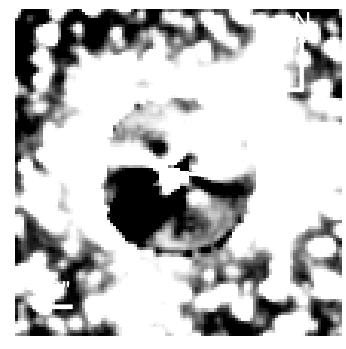

Model Sub

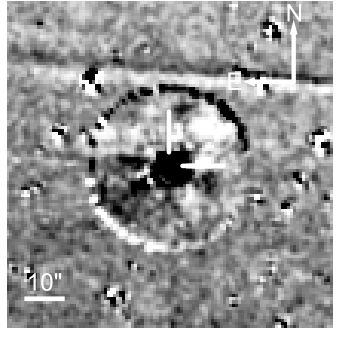

Double Sub
IC 563, Epoch 5
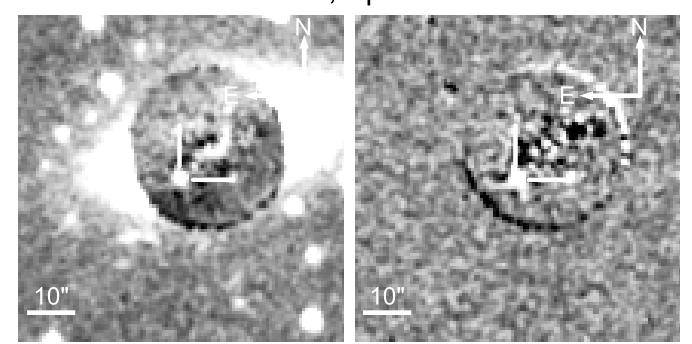

NGC 3256, Epoch 7

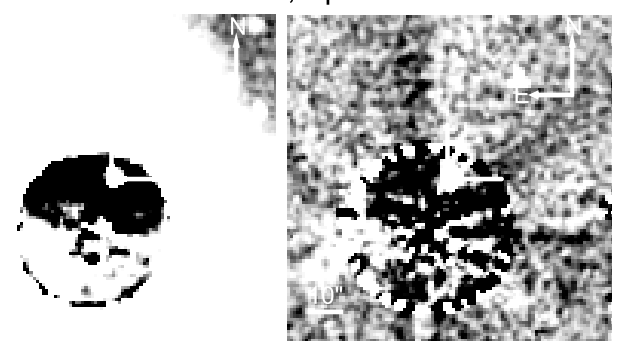

NGC 6240, Epoch 5
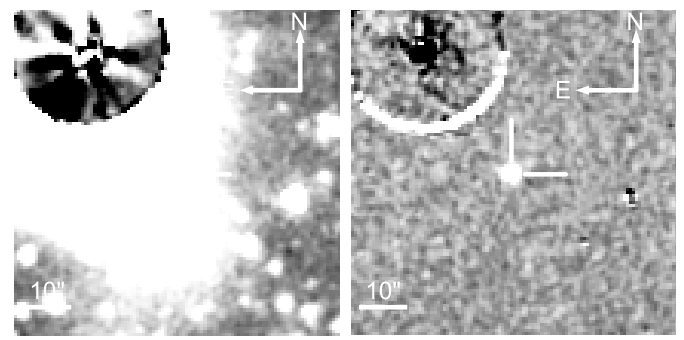

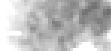
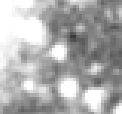

NGC 6090, Epoch 7
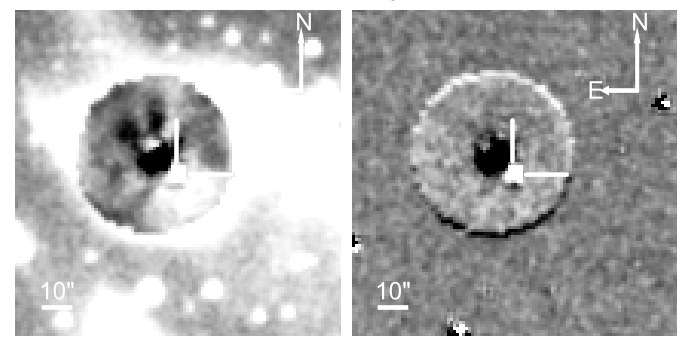

$-0.01$

Figure 9. Imaging of each SN detected in this survey, labeled by galaxy and epoch. For each detection, two images are displayed. On the left is the subtraction of the template derived using forward modeling. On the right is the subtraction of two different epochs that have been forward modeled. Epochs separated by $\sim 1 \mathrm{yr}$ have similar orientations and the relative subtractions remove additional residuals that were still visible after forward modeling, as well as the more diffuse, outer galaxy that was not forward modeled. In general, this relative subtraction of epochs separated by about a year provided optimal results. 


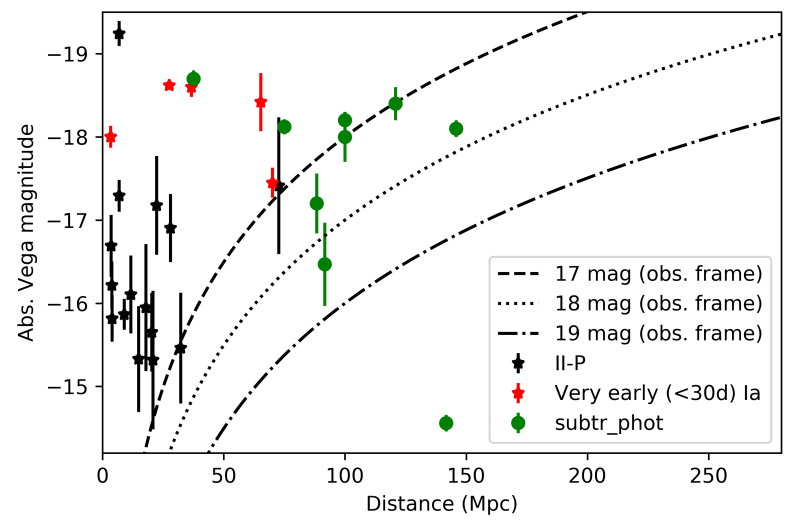

Figure 10. Absolute magnitudes of the nine SNe discovered in our sample (green symbols). The photometry corresponds to zero host galaxy extinction for all the events and therefore their absolute magnitudes should be considered as lower limits. Also plotted are early (i.e., $<3$ month) photometry from Szalai et al. (2019) of nearby (<50 Mpc) SNe IIP and some SNe Ia within 30 days of explosion (black and red symbols, respectively). Dashed, dotted, and dotted-dashed lines represent apparent brightness levels of 17, 18, and 19 mag, respectively.

SNe we detect are relatively bright. In fact, as noted in Figure 11, an absolute magnitude $M_{3.6 \mu \mathrm{m}} \approx-18$ may be interpreted as more consistent with the peak magnitudes of SNe Ia rather than those of SNe IIP. Although our sample is limited in size, we conclude that we are Malmquist biased and generally do not detect $\mathrm{SNe}$ dimmer than $17.5 \pm 0.5 \mathrm{mag}$. This empirical limit is compared to the results of our artificial-star tests in Figure 5. While in most cases the empirical threshold is brighter than the artificial-star tests achieved, there are a number of cases where the artificial-star tests are brighter than the empirical limit, particularly in the innermost regions of the galaxies. As noted in Section 3.2, there are a number of caveats in the sensitivity of the artificial-star tests to the fainter stars. In general, we therefore define our sensitivity limit for a given radial bin to be the brighter of these two limits.

\subsection{Possible Causes for Decreased Sensitivity}

This survey is not particularly sensitive to $\mathrm{SNe}$ in either the nuclear regions of galaxies or galaxies at $>150 \mathrm{Mpc}$. Cresci et al. (2007) offer some explanations for their HST/NICMOS survey that could apply to our survey. For example, the FIR flux used to calculate the expected CCSNr is dominated by obscured AGNs and not by star formation. Estimates of the relative AGN power, however, have been made using a variety of MIR tracers in hundreds of local IR galaxies and, in most cases, do not suggest significant AGN contributions (e.g., Díaz-Santos et al. 2017).

Another possibility is that the $\mathrm{SNe}$ may be more obscured by dust than originally expected, or underluminous $\mathrm{SNe}$ (e.g., Pastorello et al. 2004) form a significant fraction of all CCSNe. In the latter case, the SNe would stay above our detection limit for a shorter time. In either case, the Gaussian distribution of magnitudes would have a tail skewed toward dimmer objects and our overall sensitivity would decrease, resulting in a decrease in our expected CCSNr.

The most likely explanation, however, is a combination of worse than expected subtraction residuals combined with relatively poor resolution, despite the decreased extinction afforded by obser-

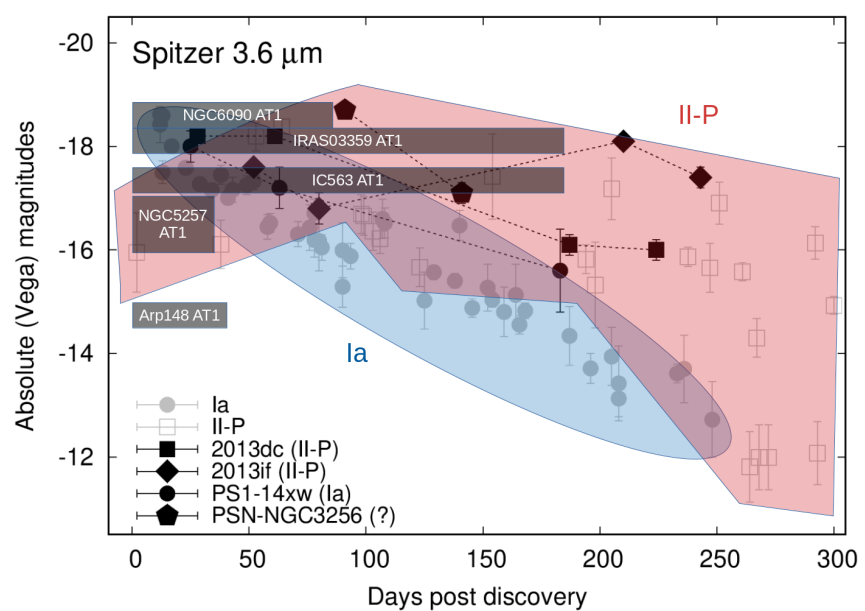

Figure 11. The $3.6 \mu \mathrm{m}$ light curves of the four detected known transients in our Survey (with black symbols), together with previously published light-curves (grey symbols and shaded colored regions, adopted from Szalai et al. 2019). Dark-grey transparent rectangles mark the single epoch of photometry for the other five detected sources within the possible periods (taking into account the dates of last non-detections). Widths and heights of the rectangles denote the uncertainties of epochs and absolute magnitudes of the five previously unknown transients, respectively.

vations at $3.6 \mu \mathrm{m}$. Note that the mosaic images have a pixel scale of $\sim 0.6^{\prime \prime}$ that corresponds to a projected distance of $\sim 290 \mathrm{pc}$ at $100 \mathrm{Mpc}$ (and the real resolution is a factor of $2-3$ worse). Our poor residual at $<3^{\prime \prime}$ corresponds to $\sim 1.5 \mathrm{kpc}$ at these distances. If most starburst activity is concentrated within the galaxy nucleus, then our survey not being sensitive to a majority of these events. We correct for the limitations in our survey sensitivity below.

\subsection{Observed vs. Expected}

To properly compare the observed number of SNe detected to the predicted value, we first have to correct for the number of expected $\mathrm{SNe}$ to account for our decreased sensitivity. To properly correct, we must calculate both the fraction of $\mathrm{SNe}$ we are sensitive to at any given location in a galaxy, $f_{\mathrm{SNe}}$, and the fraction of light within that radius, $f_{\text {light }}$.

We first define our detection threshold in Section 4.2. We then assume the distribution of SN magnitudes to be consistent with observations in Figure 2, which we take to be a Gaussian centred function at $M_{3.6 \mu \mathrm{m}}=-17 \mathrm{mag}$ and a standard deviation of $\sigma_{\text {SN IIP }}=0.7$. No extinction is applied. This distribution should be considered an upper limit as the actual function may have a lowerluminosity tail or be shifted to a somewhat less-luminous centre in the case of high extinction. For each galaxy, we calculate the respective apparent magnitude distribution for the given distance and the corresponding fraction of $\mathrm{SNe}, f_{\mathrm{SNe}}$, in the magnitude distribution that is bright enough to be detected based on the detection threshold. This fraction is calculated separately for each radial bin.

We next derive the fraction of light within each radial bin, $f_{\text {light }}$, from the integrated MIR lumninosity, which we measure using aperture photometry performed on archival IRAC and MIPS data (when available). Although the SFR and the CCSNr are tied to the FIR luminosity, we assume the MIR to be a useful and convenient proxy, at least to first order. While most (U)LIRG flux comes out at longer wavelengths (i.e., $\sim 70 \mu \mathrm{m}$ ), the shape of a (U)LIRG SED is 

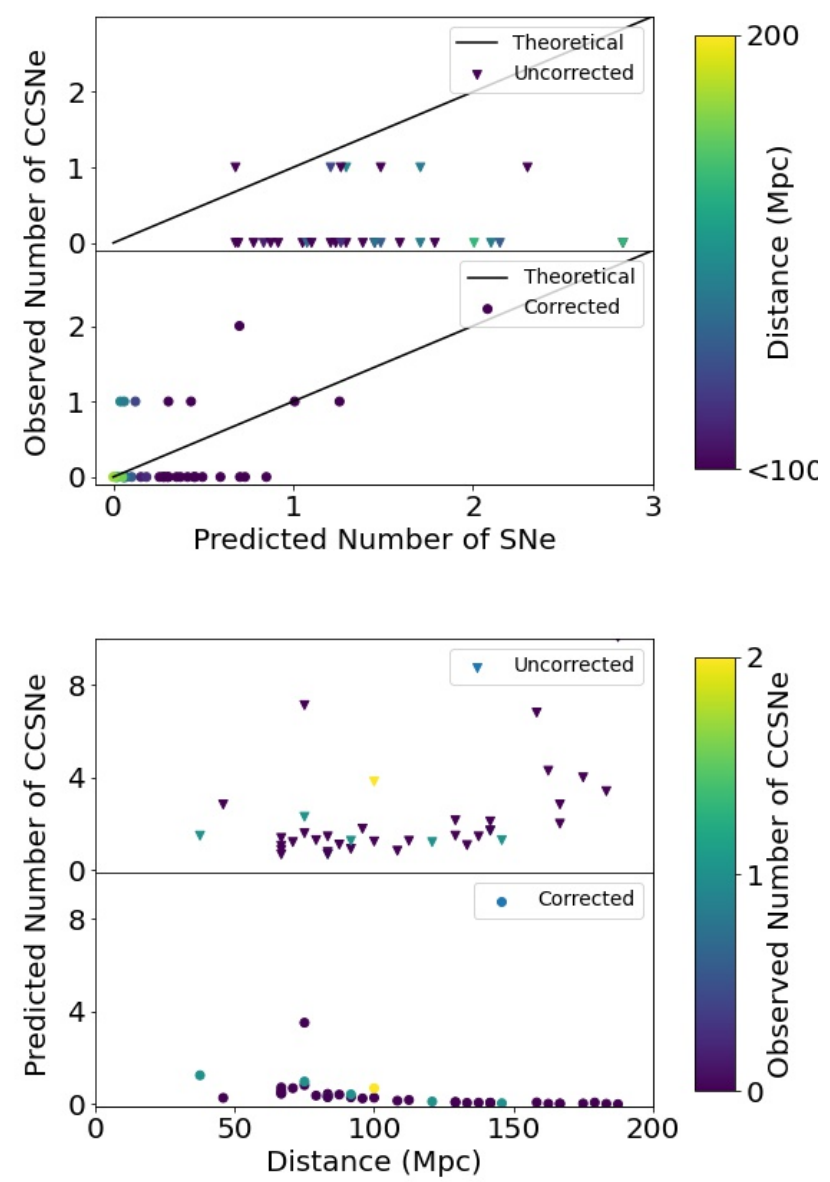

Figure 12. Relationship between predicted CCSN rate, actual CCSN rate, and distance. (Top:) The number of actual detections compared to the predicted number of CCSNe, where the colour bar corresponds to the distance (Mpc), for both the uncorrected and corrected rates. (Bottom:) The predicted number of $\mathrm{SNe}$ as a function of distance, where the colour bar corresponds to the number of actual detections. Both plots show values for both the uncorrected and corrected rates (as described in the text).

roughly constant (Wright et al. 2014). Table 3 lists our derived MIR luminosities and fractional luminosity for each aperture.

The predicted CCSNr is finally corrected by multiplying by both the fraction of $\mathrm{SNe}$ we are sensitive to, $f_{\mathrm{SNe}}$, and the fraction of light within that radial bin, $f_{\text {light }}$. Table 1 lists the corrected CCSNr predictions, and Figure 12 compares the predicted and observed number of SNe detected for each galaxy. Figure 12(a) compares the number of observed CCSNe to the number of predicted CCSNe. Overplotted is a line corresponding to a 1:1 ratio, which is what we would expect in an ideal scenario. The two plots correspond to the uncorrected and corrected number of predicted events, respectively. Figure 12(b) plots the predicted number of CCSNe as a function of distance. Again, the upper and lower panels correspond to the uncorrected and corrected number of predicted events, respectively.

There are several qualitative takeaways. First, all of our detections occur at $<150 \mathrm{Mpc}$. Although our calculations suggest that we should be sensitive to $\mathrm{SNe}$ out to $200 \mathrm{Mpc}$, other challenges arise at these distances. For example, galaxies become less resolved and more compact, so the subtraction residuals have a larger im-
Table 3. IR Integrated Luminosities (5-24 $\mu \mathrm{m})$

\begin{tabular}{|c|c|c|c|c|}
\hline \multirow{2}{*}{$\begin{array}{l}\text { Galaxy } \\
\text { Name }\end{array}$} & $1^{\prime \prime}$ & & & \\
\hline & \multicolumn{4}{|c|}{$\mathrm{L}_{\odot}$ (Fraction of Total Luminosity) } \\
\hline NGC034 & $9.7(0.1)$ & $10.4(0.47)$ & $10.5(0.62)$ & $10.7(1.0)$ \\
\hline NGC232 & $3(0.05)$ & & & $10.6(1.0)$ \\
\hline MCG+12-02 & $2(0.04)$ & $10.0(0.24)$ & $10.3(0.47)$ & $10.7(1.0)$ \\
\hline IC1623 & $8.9(0.02)$ & $9.8(0.15)$ & $10.1(0.32)$ & $10.6(1.0)$ \\
\hline UGC2369 & $9.0(0.02)$ & $6(0.11)$ & $10.0(0.25)$ & $0.6(1.0)$ \\
\hline IRAS03359 & $9.7(0.07)$ & & & $10.8(1.0)$ \\
\hline MCG-03- & & & & $6(1.0)$ \\
\hline & $0.09)$ & & & $.5(1.0)$ \\
\hline NGC1 & & & & $10.8(1.0)$ \\
\hline NGC & & & & $10.6(1.0)$ \\
\hline & & & & $0.9(1.0)$ \\
\hline UGC & & 10.8 & 10. & $11.1(1.0)$ \\
\hline MCG & & 6) & & $9.8(1.0)$ \\
\hline IC563 & & 2) & $9.8(0.36)$ & $10.3(1.0)$ \\
\hline NGC3110 & $1(0.05)$ & $9.9(C$ & $10.1(0.46)$ & $10.4(1.0)$ \\
\hline NGC3256 & $3(0.04)$ & $10.1(0$ & 10.4 & $10.7(1.0)$ \\
\hline IRAS1C & $.2(0.1)$ & 10.8 & 11.0 & $11.2(1.0)$ \\
\hline Arp1 & 8) & & & $10.8(1.0)$ \\
\hline MCG+00-29 & 09) & 10.3 & 10. & $10.6(1.0)$ \\
\hline IC2810 & & & & $10.8(1.0)$ \\
\hline & & & & \\
\hline ESO5 & & & 10.3 & $10.6(1.0)$ \\
\hline & & & & $9.8(1.0)$ \\
\hline & & 10.4 & 10. & 10.8( \\
\hline NGC & 2) & & 10.2 & $10.7(1.0)$ \\
\hline NGC & 1) & & 2) & $10.4(1.0)$ \\
\hline Mk27 & $10.4(0.09)$ & 11.0 & 11.1 & $11.4(1.0)$ \\
\hline NGC5 & $.06)$ & $10.3(0.32)$ & $10.5(0.47)$ & $10.8(1.0)$ \\
\hline UGC8782 & $3(0.08)$ & $9.9(0.35)$ & $10.1(0.48)$ & $10.4(1.0)$ \\
\hline Arp302 & $9.7(0.06)$ & $10.4(0.3)$ & $10.6(0.48)$ & $10.9(1.0)$ \\
\hline Mk84 & $0.0(0.1)$ & $10.7(0.41)$ & $10.8(0.54)$ & $11.1(1.0)$ \\
\hline Arp220 & $9.8(0.15)$ & $10.4(0.69)$ & $10.5(0.83)$ & $10.6(1.0)$ \\
\hline NGC6090 & $9.1(0.02)$ & $9.9(0.14)$ & $10.2(0.3)$ & $10.7(1.0)$ \\
\hline NGC6240 & $10.0(0.11)$ & $10.7(0.47)$ & $10.8(0.6)$ & $11.0(1.0)$ \\
\hline IRAS1720 & $10.4(0.08)$ & $1.0(0.35)$ & $11.2(0.49)$ & $11.5(1.0)$ \\
\hline IC4687 & $(0.05)$ & $10.1(0.34)$ & $10.4(0.55)$ & $10.6(1.0)$ \\
\hline IRAS18293 & $9.7(0.06)$ & $10.5(0.32)$ & $10.7(0.5)$ & $11.0(1.0)$ \\
\hline NGC6926 & $8(0.04)$ & $9.5(0.18)$ & $9.7(0.32)$ & $10.2(1.0)$ \\
\hline NGC7130 & $9.4(0.08)$ & $10.1(0.34)$ & $10.2(0.49)$ & $10.5(1.0)$ \\
\hline IRAS23128 & $10.1(0.09)$ & $10.8(0.38)$ & $10.9(0.54)$ & $11.2(1.0)$ \\
\hline
\end{tabular}

pact. These residuals correspond to larger projected distances in, and therefore fraction of, the host galaxy. While there is no sharp cutoff, the more distant galaxies in our sample are more luminous, but also more compact and dustier.

Second, in almost every case the adjusted expected $\mathrm{CCSNr}$ ends up falling to $<1$, which makes a comparison to the expected trend (black line) in Figure 12(a) difficult. Despite small-number statistics, we still see a slight correlation between observed SNe versus predicted $\mathrm{SNe}$ that, although weak, does provide a useful self-consistency check. The one exception is Arp 220, which stands out from the other galaxies at $\sim 75 \mathrm{Mpc}$ for its large number of predicted SNe. Despite this expectation, we don't detect any $\mathrm{SNe}$, possibly due to the fact that most of the light is concentrated in the inner $<3^{\prime \prime}$. Furthermore, like other IR-bright galaxies, Arp 220 may suffer from relatively high local extinction.

Taken all together, these adjustments limit a significant fraction of the phase space in which we can search for and detect an SN. The large number of galaxies observed, however, compensates for these inefficiencies and provides adequate statistics. In total, we expect 96 and $14 \pm 6 \mathrm{SNe}$ before and after corrections are made (respectively), 
where the error bars are dominated by uncertainty assigned above to our detection threshold (i.e., $m_{3.6 \mu \mathrm{m}}=17.5 \pm 0.5 \mathrm{mag}$ ). We discover $9 \mathrm{SNe}$ (Table 2).

\subsection{Statistical Implications}

After all corrections are taken into account, our observations yield a statistically significant sample that will enable us to differentiate between (1) undetected $\mathrm{SNe}$ and (2) counting statistics. Assuming the counting statistics are determined by the SN sample (i.e., $\sigma=$ $\sqrt{N}$ ), then the minimum number of SNe we must expect to detect is roughly sixteen. Any fewer expected $\mathrm{SNe}$ would be statistically insufficient. For example, to identify a $3 \sigma$ deficit in the CCSNr when only $9 \mathrm{SNe}$ are expected, one would need to observe $<0 \mathrm{SNe}$.

As noted above, we detect $9 \mathrm{SNe}$, whereas theory predicted $14 \pm 6 \mathrm{SNe}$. We conclude that our observations are generally consistent with the predictions, especially when considering the counting statistics, which for $14 \mathrm{SNe}$ yield $\sigma_{\text {counting }}=\sqrt{14}=3.7$. Of course, the number of predicted $\mathrm{SNe}$ is given after significant corrections in our sensitivity have been applied.

\section{CONCLUSION}

We have presented a Spitzer/IRAC MIR survey for dustextinguished $\mathrm{SNe}$ to resolve the discrepancy between theoretically predicted and optical/NIR observed core-collapse SN rates. We searched 40 nearby $(<200 \mathrm{Mpc})$ star-forming galaxies using Spitzer/IRAC. The survey includes eight epochs of observations obtained in the years 2012-2014.

Our ability to detect new SNe was dominated by subtraction residuals near the nuclear regions $\left(<3{ }^{\prime \prime}\right)$ of each galaxy caused by an asymmetric PRF that rotated throughout the year as the telescope circled the Sun. To optimise our subtraction algorithm, we implemented a forward modeling technique that will be employed on the NGRST; (Rubin et al. 2021).

While forward modeling improves our overall sensitivity, the number of discovered $\mathrm{SNe}$ is still substantially lower than the expected intrinsic number during the survey period. We compensate for the number of predicted SNe by quantifying the inefficiencies, mostly arising from remaining residuals in the inner $<3^{\prime \prime}$, where a majority of star formation occurs. The predicted rates are particularly impacted by the fact that most of the galaxies with the highest $\mathrm{SN}$ rates (2-11 SNe per year) are located at distances larger than $150 \mathrm{Mpc}$, where essentially zero SNe can be expected to be discovered owing to the sensitivity limits of the search (Table 1). After all corrections are applied, we expect to discover $14 \pm 6 \mathrm{SNe}$ and actually find $9 \mathrm{SNe}$, which suggests that our observations are consistent with the theoretical expectations.

While still hampered by high extinction, if most starburst activity is concentrated within the inner nucleus of the galaxy (for this experiment $<1.5 \mathrm{kpc}$ at $100 \mathrm{Mpc}$ ), a survey with both higher resolution and longer-wavelength observations is necessary. A stable, space-based PSF is needed, too. Figure 1 shows that the optimal wavelength is actually closer to $2.7 \mu \mathrm{m}$. With the upcoming launch of JWST, with a PSF of $\sim 0.091^{\prime \prime}$ in its F277W filter, such a survey to probe the nuclear regions of star-forming galaxies for transients will finally be possible.

\section{ACKNOWLEDGEMENTS}

The authors acknowledge an anonymous referee for helpful comments that led to improvements in this paper. We also give tremendous thanks to the IRAC instrument support team at the Spitzer Science Center (SSC) for their continued assistance over the years. They are also grateful to Rick Arendt for his helpful comments. O.D.F. additionally thanks Peter Capak, Sean Carey, and Patrick Lowrance for useful technical discussions. This work is based on observations made with the Spitzer Space Telescope (PID 90031), which is operated by the Jet Propulsion Laboratory, California Institute of Technology, under a contract with NASA. Support for this work was provided by NASA through an award issued by JPL/Caltech, primarily supporting students C.C., G.L., and H.K. Additional support was provided to D.R. and H.K. by STScI's DDRF Grant D0001.82477 and the TSRC program. T.S. is supported by the János Bolyai Research Scholarship of the Hungarian Academy of Sciences, by the New National Excellence Program (UNKP-20-5) of the Ministry of Technology and Innovation of Hungary, by the GINOP-2-3-2-152016-00033 project of the National Research, Development, and Innovation Office of Hungary (NKFIH) funded by the European Union, and by NKFIH/OTKA FK-134432 grant. A.V.F. acknowledges support from the TABASGO Foundation, the Christopher R. Redlich Fund, and the U.C. Berkeley Miller Institute for Basic Research in Science (in which he is a Senior Miller Fellow).

\section{Data availability}

The data underlying this article will be shared on reasonable request to the corresponding author.

\section{REFERENCES}

Benitez S., et al., 2014, The Astronomer's Telegram, 6107, 1

Block A., et al., 2013, Central Bureau Electronic Telegrams, 3551, 1

Bregman J. D., Temi P., Rank D., 2000, A\&A, 355, 525

Chary R., Elbaz D., 2001, ApJ, 556, 562

Cresci G., Mannucci F., Valle M. D., Maiolino R., 2007, A\&A, 462, 927

Dahlén T., Fransson C., 1999, A\&A, 350, 349

Dahlen T., et al., 2004, The Astrophysical Journal, 613, 189

Díaz-Santos T., et al., 2017, ApJ, 846, 32

Fazio G. G., et al., 2004, ApJS, 154, 10

Filippenko A. V., 1997, ARA\&A, 35, 309

Floc'h E. L., et al., 2005, The Astrophysical Journal, 632, 169

Genzel R., et al., 1998, Astrophysical Journal v.498, 498, 579

Grossan B., Spillar E., Tripp R., Pirzkal N., Sutin B. M., Johnson P., Barnaby D., 1999, The Astronomical Journal, 118, 705

Hayden B., et al., 2021, arXiv e-prints, p. arXiv:2103.13285

Holtzman J. A., et al., 2008, AJ, 136, 2306

Iben I., 1983, Astrophysical Journal, 275, L65

Jencson J. E., et al., 2019, ApJ, 886, 40

Kankare E., et al., 2008, The Astrophysical Journal, 689, L97

Kankare E., et al., 2012, The Astrophysical Journal Letters, 744, L19

Kankare E., et al., 2021, A\&A, 649, A134

Kasliwal M. M., et al., 2017, ApJ, 839, 88

Kennicutt Robert C. J., 1998, ARA\&A, 36, 189

Kool E. C., et al., 2018, MNRAS, 473, 5641

Lawrence C., Gautier N., 2004, Spitzer Proposal ID \#108, p. 108

Madau P., Dickinson M., 2014, ARA\&A, 52, 415

Magnelli B., Elbaz D., Chary R. R., Dickinson M., Borgne D. L., Frayer D. T., Willmer C. N. A., 2009, Astronomy and Astrophysics, 496, 57

Magnelli B., Elbaz D., Chary R. R., Dickinson M., Borgne D. L., Frayer D. T., Willmer C. N. A., 2011, Astronomy \& Astrophysics, 528, 35 
Maiolino R., Vanzi L., Mannucci F., Cresci G., Ghinassi F., Valle M. D., 2002, Astronomy and Astrophysics, 389, 84

Maiolino R., Oliva E., Ghinassi F., Pedani M., Mannucci F., Mujica R., Juarez Y., 2004a, Astronomy and Astrophysics, 420, 889

Maiolino R., Schneider R., Oliva E., Bianchi S., Ferrara A., Mannucci F., Pedani M., Sogorb M. R., 2004b, Nature, 431, 533

Mannucci F., et al., 2003, A\&A, 401, 519

Mannucci F., Valle M. D., Panagia N., 2007, Monthly Notices of the Royal Astronomical Society, 377, 1229

Matteucci F., Panagia N., Pipino A., Mannucci F., Recchi S., Valle M. D., 2006, Monthly Notices of the Royal Astronomical Society, 372, 265

Mattila S., Meikle W. P. S., 2001, Monthly Notices RAS, 324, 325

Mattila S., Meikle W. P. S., Greimel R., 2004, New Astron. Rev., 48, 595

Mattila S., Monard L. A. G., Li W., 2005a, IAU Circ., 8473, 2

Mattila S., et al., 2005b, IAU Circ., 8474,

Mattila S., et al., 2007, The Astrophysical Journal, 659, L9

Mattila S., et al., 2012, ApJ, 756, 111

Miluzio M., et al., 2013, A\&A, 554, A127

Navasardyan H., Petrosian A. R., Turatto M., Cappellaro E., Boulesteix J., 2001, Monthly Notices of the Royal Astronomical Society, 328, 1181

Pastorello A., et al., 2004, MNRAS, 347, 74

Pérez-Torres M., Mattila S., Alonso-Herrero A., Aalto S., Efstathiou A., 2021, A\&ARv, 29, 2

Rho J., Reach W. T., Tappe A., Hwang U., Slavin J. D., Kozasa T., Dunne L., 2009, eprint arXiv, 0905, 497

Richmond M. W., Filippenko A. V., Galisky J., 1998, The Publications of the Astronomical Society of the Pacific, 110, 553

Rubin D., Cikota A., Aldering G., Fruchter A., Perlmutter S., Sako M., 2021, arXiv e-prints, p. arXiv:2102.05069

Sanders D. B., Mirabel I. F., 1996, Annual Review of Astronomy and Astrophysics, 34, 749

Sanders D. B., Mazzarella J. M., Kim D.-C., Surace J. A., Soifer B. T., 2003, The Astronomical Journal, 126, 1607

Scannapieco E., Bildsten L., 2005, The Astrophysical Journal, 629, L85

Smith N., Li W., Filippenko A. V., Chornock R., 2011, Monthly Notices of the Royal Astronomical Society, 412, 1522

Strolger L.-G., et al., 2015, ApJ, 813, 93

Suzuki N., et al., 2012, ApJ, 746, 85

Szalai T., Zsíros S., Fox O. D., Pejcha O., Müller T., 2019, ApJS, 241, 38

Wright J. T., Griffith R. L., Sigurdsson S., Povich M. S., Mullan B., 2014, ApJ, 792, 27

van Buren D., Greenhouse M. A., 1994, The Astrophysical Journal, 431, 640 